\title{
A label-free method for studying DNA sequence recognition of mitoxantrone based on resonance light-scattering technique
}

\author{
Zhanguang Chen ${ }^{1}$, Guomin Zhang ${ }^{1}$, Xi Chen ${ }^{2}$, Junhui Chen ${ }^{3}$ and Jinbin Liu ${ }^{4}$
}

A label-free method for studying DNA sequence recognition of mitoxantrone (MIT) by resonance light-scattering (RLS) technique has been developed in this contribution. Through the RLS spectra, the selective no-covalent interactions between MIT and double-stranded DNA, single-stranded DNA (ssDNA), oligonucleotides were systematically studied. The results of the experiments displayed that MIT had an obvious preference to SSDNA with the $K\left(K_{\mathrm{RLS}}\right), 15.16 \mathrm{mmol} \mathrm{mg}^{-1}$ and the number of binding sites $(N), 8.68 \times 10^{-4} \mathrm{mmol} \mathrm{mg}^{-1}$. Besides, it was found that MIT had a preference to sequences that were rich in guanine and cytosine bases with $K_{\mathrm{RLS}}, 17.29 \mathrm{Immol}^{-1}$ and $N, 1.19 \times 10^{-2} / \mathrm{mmol}^{-1}$. The recognition mechanisms were well discussed and by fluorescence method and atom force microscopy, the RLS technique was confirmed to be a reliable method in this study. Compared with other methods, what the RLS strategy displayed was the direct interaction between anticancer drugs and DNA in vitro without the influence of a foreign substance. Thus, it can be a simple, fast and label-free strategy for DNA sequence recognition studies of DNA-targeted anticancer drugs.

The Journal of Antibiotics (2012) 65, 517-522; doi:10.1038/ja.2012.58; published online 18 July 2012

Keywords: anticancer drugs; mitoxantrone; oligonucleotides; recognition; resonance light scattering; selectivity

\section{INTRODUCTION}

Owing to the need of cancer therapy and anticancer drugs design, investigation to the interaction between DNA and DNA-targeted anticancer drugs (DTAD) is increasingly significant. ${ }^{1}$ Studying their micro-mechanisms, such as binding mode, DNA sequence recognition and structure-activity relationships, can not only display their identification and assembling mechanism detailedly, but also supply a theoretical basis for anticancer drug design. ${ }^{2-7}$ As is well known, the toxicity or side effect of anticancer drugs is generally generated by their attacking to the normal human cells. ${ }^{8}$ With the aim to decrease it, researches on the selectivity of anticancer drugs have been of more and more significance. ${ }^{9}$ Among these researches, the investigation on the sequence recognition of DTAD has been a basis for others..$^{10}$ At present, there are some main methods of investigation on the sequence recognition of DTAD, such as NMR assay, ${ }^{11} \mathrm{X}$-ray assay, ${ }^{12}$ and theoretical modeling assay. ${ }^{13}$ But the equipments of these methods are expensive with the experimental procedures too complex. To make up for these disadvantages, chemiluminescence assays $^{14}$ and electrochemical methods, ${ }^{15}$ in which fluorescence regents, conjugated polymers and nanomaterials are always used as probes, have been gradually developed recently. Owing to their advantages of simplicity and low cost, serial similar assays for studying the selectivity of anticancer drugs have been proposed.
But what should be noted is that a foreign reagent always has to be used as the probe in these methods and thus the weak interaction between DTAD and DNA will be influenced easily. So the results of these methods may not be what the fact is. In order to overcome the above problem, we firstly developed a resonance light-scattering (RLS) strategy to non-damagingly investigate the DNA sequence selectivity of anticancer drugs and their selectivity mechanism in this contribution.

RLS is a kind of elastic scattering. The successful application of it in analytical chemistry was firstly reported in 1993, in which Pasternack et al. ${ }^{16,17}$ successfully studied the J-assembly of trans-bis ( $N$-methylpyridinium-4-y1) diphenylporphine and its copper(II) derivative on DNA using a common fluorescence spectrophotometer. In 1996, Huang et al. ${ }^{18,19}$ brought RLS technique into the quantitative analytical chemistry and completed the determination of DNA. Subsequently, RLS technique has obtained a wide application in analytical chemistry because of its simplicity, low cost and reliability. ${ }^{20-25}$ In our lab, this technique has been successfully used in the environmental analysis ${ }^{26}$ and DTAD screening. ${ }^{27-30}$

Mitoxantrone (MIT, shown in Supplementary Figure S1), as a kind of widely used anticancer drugs, has a similar structure with doxorubicin. It can insert into the duplex of DNA and form a

${ }^{1}$ Department of Chemistry, Shantou University, Shantou, China; ${ }^{2}$ Guangdong Pharmaceutical University, Guangzhou, China; ${ }^{3}$ Department of Radiology, Peking University Shenzhen Hospital, Shenzhen, China and ${ }^{4}$ Department of Chemistry, University of Texas at Dallas, Richardson, TX, USA 
complex, which can restrain the activity of polymerases of DNA and RNA, which is also the basis of its antitumor effect. ${ }^{31}$ In this contribution, the selective non-covalent interaction between MIT and DNA has been investigated non-damagingly by the RLS strategy; especially the DNA sequence recognition of MIT was systematically studied and the selective mechanism was also discussed. In order to confirm the reliability of the RLS assay, atomic force microscope (AFM, Digital Instruments, Santa Barbara, CA, USA) and fluorescence method were also involved and the results confirmed that the RLS technique can be an effective and reliable method in the research of selectivity of anticancer drugs.

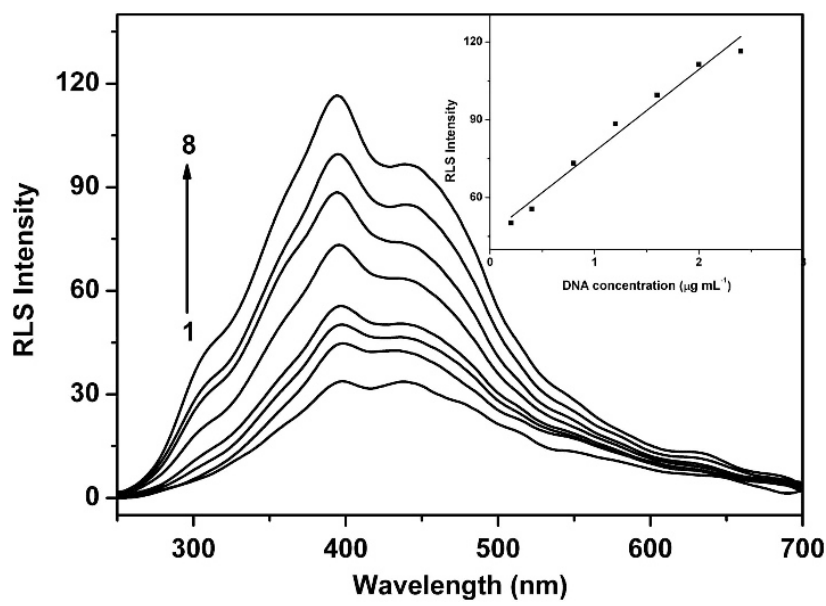

Figure 1 The RLS spectrum of MIT-dsDNA. (The inset is the relationship between the RLS intensity and concentration of dsDNA) Conditions: $\mathrm{pH}=2.0, \mathrm{MIT}, 1.0 \mu \mathrm{gml} \mathrm{m}^{-1}$, dsDNA $\left(\mu \mathrm{gml} \mathrm{ml}^{-1}\right)$ : 1-0, 2-2.0 (without MIT), 3-0.2, 4-0.4, 5-0.8, 6-1.2, 7-1.6, 8-2.4.

a

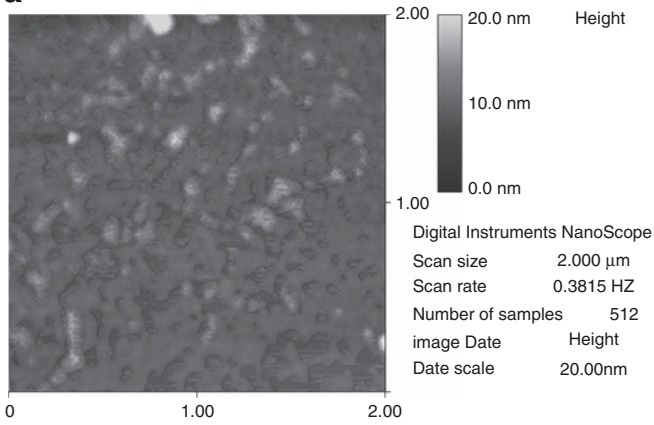

C

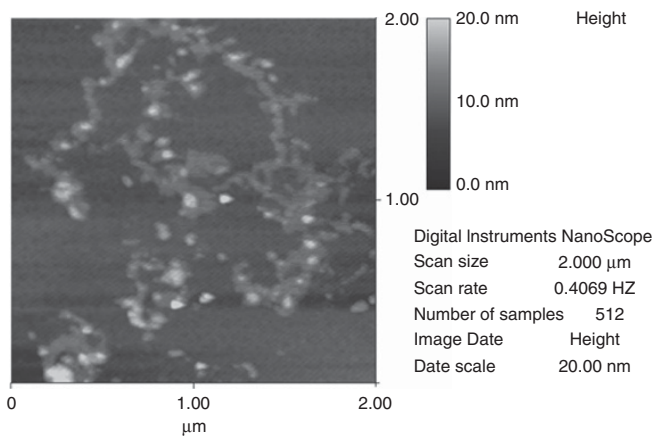

\section{RESULTS AND DISCUSSION}

The RLS spectra of MIT-double-stranded DNA (dsDNA) under optimum conditions was shown in Figure 1, from which we can see that the RLS signals of MIT and dsDNA at $395 \mathrm{~nm}$ were both very weak. Although certain amount of dsDNA was added into the MIT solutions, the RLS signal enhanced dramatically and it had a good linear relationship with the concentration of dsDNA $(Y=46.10$ $+31.64 X)$. According to what has been reported, the increment of RLS signals at $395 \mathrm{~nm}$ was attributed to the MIT-dsDNA complex, which was formed by the insertion of MIT molecule into the duplex of dsDNA. ${ }^{31}$ Meanwhile, the hydrogen bond between the electronegative groups of MIT and dsDNA can also stabilize the MIT-dsDNA complex, ${ }^{32}$ which also resulted in the remarkable enhancement of RLS signals. Based on the phenomenon above, we exercised the advantage of high sensitivity of RLS technique in the nucleotides research in vitro and brought it into different sequence systems. By comparing their preferences to different sequences, which are obtained from the RLS spectra, DNA sequence recognition of DTAD can be studied non-damagingly. In this strategy, it doesn't need any probe and what it displays is the real direct interaction between DTAD and different DNA sequences in vitro.

Figure 2 is the AFM schemes of dsDNA and MIT-dsDNA complex. Obviously, it can be seen that dsDNA without MIT is mainly in the presence of dispersive particles, which are formed by the hydrophobic effect of dsDNA. After MIT is added, it's clearer in the threedimensional schemes that dsDNA assembles into large particles and intertwines together. So it's the assembling and intertwining of dsDNA that induce the increase of RLS intensity of MIT-dsDNA system. Moreover, the fluorescence spectra of MIT-dsDNA system were studied. As displayed in Figure 3, the fluorescence intensity of the system gradually decreased with the increase of dsDNA concentration. When the dsDNA concentration was $8.0 \mu \mathrm{g} \mathrm{ml}^{-1}$, the b

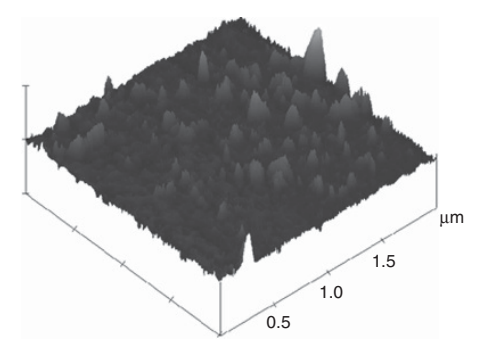

d

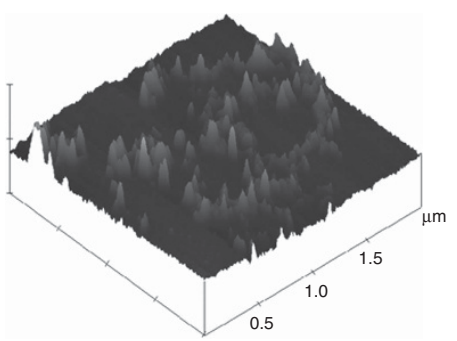

Figure 2 AFM schemes of dsDNA $\left(1.0 \mu \mathrm{g} \mathrm{ml}^{-1}\right.$ ) at presence of MIT $\left(\mu \mathrm{g} \mathrm{ml} \mathrm{I}^{-1}\right)$ : (a) 0 ; (b) 0; (c) 1.0; (d) 1.0. (a, c is the two-dimensional AFM schemes of dsDNA and MIT-dsDNA complex, respectively, and $(\mathbf{b}, \mathbf{d})$ is the three-dimensional AFM schemes of dsDNA and MIT-dsDNA complex, respectively). 


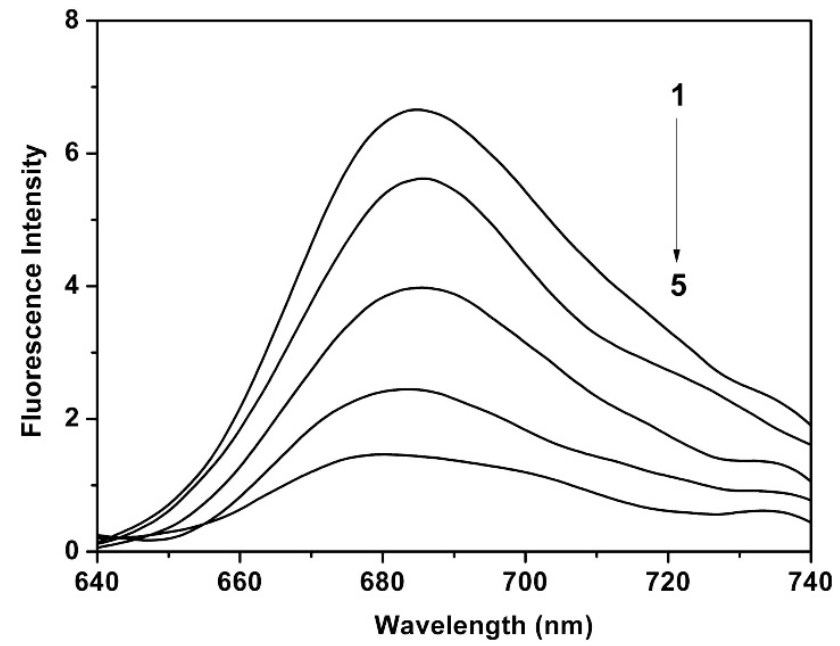

Figure 3 Fluorescence spectrum of MIT-dsDNA. Conditions: $\mathrm{pH}=2.0, \mathrm{MIT}$, $5.0 \mu \mathrm{g} \mathrm{ml}^{-1}$, dsDNA (1-5), 0, 2.0, 4.0, 6.0, $8.0 \mu \mathrm{g} \mathrm{ml}^{-1}$.

fluorescence of the system hardly quenched, which is because that anthraquinone ring structures of MIT were good electron acceptors and DNA bases were good electron donors. It is the electron transition between DNA bases and anthraquinone ring structures of MIT that induced the sharply decreased fluorescence of MIT-dsDNA system. ${ }^{33}$ Therefore, it can be confirmed that MIT can interact with dsDNA in vitro directly and it's also their interaction that results in the changing of RLS signals. Thus, the strategy is reliable to be used to non-damagingly study the DNA sequence recognition of DTAD.

In order to improve the sensitivity of RLS technique in this analytical system, the main influence factors were optimized with the dsDNA system as a model (Supplementary Figure S2). From what was shown in the figure, we can see that $\Delta I_{\text {RLS }}$ (The enhanced RLS intensity was presented as $\Delta I_{\mathrm{RLS}}=I_{\mathrm{RLS}}-I_{\mathrm{RLS}}^{0}$, Where, $I_{\mathrm{RLS}}$ and $I^{0}{ }_{\mathrm{RLS}}$ were the RLS intensities with and without MIT, respectively.) gradually decreased with the increase of $\mathrm{pH}$. And thus $\mathrm{pH}=2.0$ was selected as the optimized acid condition in this work. As to this phenomenon, we considered that the hydroxyls and amino of MIT would be in the form of positive ions under low $\mathrm{pH}$ and these positive ions could attack the phosphate skeleton of dsDNA. So the complex of MIT-dsDNA under low $\mathrm{pH}$ was more stable and the RLS signal was stronger. Similarly, the RLS signals increased gradually with the increase of concentration of MIT in a relative range and when the concentration of MIT was $1.0 \mu \mathrm{g} \mathrm{ml}^{-1}$, the RLS intensity reached the highest. But the RLS signals of the system began to decrease while the concentration of MIT was higher than $1.0 \mu \mathrm{g} \mathrm{ml}^{-1}$. This is because when the concentration of MIT was $1.0 \mu \mathrm{g} \mathrm{ml}^{-1}$, the reaction between MIT and dsDNA was completed accomplishedly. If excess MIT was added, it would affect the interaction between MIT and dsDNA, which also resulted in the decrease of the RLS intensity of the system. Therefore, $1.0 \mu \mathrm{g} \mathrm{ml}^{-1}$ was selected as the optimizing concentration of MIT.

Additionally, the influence of ion strength, effect of the incubation time and temperature were also investigated and we got the results that no other electrolytes were needed to adjust the ion intensity in this work. And the RLS intensity was stable when the temperature was kept in the range $20-40^{\circ} \mathrm{C}$. At room temperature MIT could react with dsDNA immediately after mixing together. Thus, this method does not require special crucial timing and temperature conditions. All the experiments were carried out under room temperature. In the

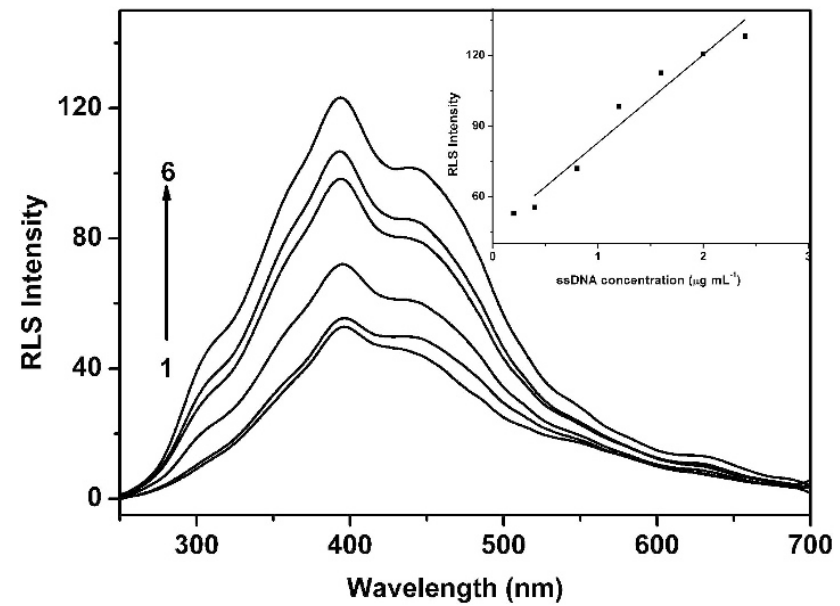

Figure 4 The RLS spectrum of MIT-SSDNA. (The inset is the relationship between the RLS intensity and concentration of SSDNA) Conditions: $\mathrm{pH}=2.0, \mathrm{MIT}, 1.0 \mu \mathrm{g} \mathrm{ml}^{-1}$, ssDNA $\left(\mu \mathrm{g} \mathrm{ml}^{-1}\right): 1-0.2,2-0.4,3-0.8,4-1.2$, $5-1.6,6-2.0$.

experiments, we have compared the results under the optimum conditions with those under physiological conditions and the results displayed they were consistent. But under the optimized conditions, the RLS intensity was much stronger and that would improve the stability and sensitivity of the method. So all the experiments were completed under the optimum conditions.

With the same strategy, into MIT solution were added certain amount of single-stranded DNA (ssDNA) solutions under the same conditions, the changes of the RLS intensity was shown in Figure 4. It's also clear that the RLS intensity gradually increased with the increase of concentration of ssDNA $(Y=46.10+31.64 X)$. In the linear ranges of dsDNA and ssDNA systems, the Rayleigh scattering equation could describe the relationships between various physical parameters and it was shown as follows: ${ }^{34}$

$$
I=I_{0} \frac{8000(2.303)^{2} \cdot \pi V n^{2}}{3 \lambda_{0}^{4} N_{\mathrm{A}}}\left\{\left[\frac{1}{\pi} \int_{0}^{\infty} \frac{\varepsilon(\lambda) d \lambda}{\lambda_{0}^{2}-\lambda^{2}}\right]^{2}+\frac{\varepsilon^{2}\left(\lambda_{0}\right)}{4 \lambda_{0}^{2}}\right\} c
$$

Where, $I_{0}$ is the intensity of incident light and $I$ is the Raleigh scattering intensity; $n$ is the refractive indices of the medium; $c$ is the concentration of particles; $V$ is the volume of the particle; $N_{\mathrm{A}}$ is the Avogadro constant; and $\lambda, \lambda_{0}$ are the wavelength of emergent and incident light, respectively. Under the same experiment conditions, these parameters $\left(V, N, \lambda, \lambda_{0}\right)$ are constants. So the scattering intensity $I_{\mathrm{RLS}}$ is proportional to the concentration of $\operatorname{drug} c$ or the number of particles in the unit volume $v$. The formula can be rewritten as below:

$$
I_{\mathrm{RLS}}=k c
$$

Herein, $[S]$ is defined as the dissociative binding site of DNA without MIT molecules; $[L]$ is defined as the equilibrium concentration of MIT, which has not reacted with DNA and $N$ is defined as the average binding sites of DNA. Then, the reaction between MIT and DNA can be described like this:

$$
[S]+[L] \stackrel{K_{\mathrm{RLS}}}{\longrightarrow}[S L]
$$

So the $K$ of above reaction is:

$$
K_{\mathrm{RLS}}=\frac{[S L]}{[S][L]}=\frac{[S L]}{\left(\mathrm{NC}_{\mathrm{DNA}}-[S L]\right)[L]}
$$


As to equation (2), it can be simplified as equation (5) because of the fact that the RLS signal is mainly resulted from the complex of MIT-DNA.

$$
\Delta I_{\mathrm{RLS}}=k[S L]
$$

$K$ can be obtained from the linear equations above and then [SL] can be figured out according to equation (5). Moreover, MIT interacts with DNA mainly by inserting and hydrogen bond, so the reaction can be characterized by the Langmuir adsorption isotherm theory:

$$
\frac{1}{n}=\frac{C_{\mathrm{DNA}}}{[S L]}=\frac{1}{K_{\mathrm{RLS}} N} \cdot \frac{1}{[L]}+\frac{1}{N}
$$

Where, $n$ is the average number of MIT molecules binding to per DNA molecule and from the equation, we can conclude that $n$ and $[L]$ are changeable with the changing of concentration of DNA. Supplementary Figure S3 displayed the relationship between $1 /[L]$ and $1 / n$, from which $N$ and $K_{\text {RLS }}$ could be figured out. The results of $N$ and $K_{\text {RLS }}$ were shown in Table 1 , from which we can see that it has a smaller $N$ but larger $K_{\mathrm{RLS}}$ in ssDNA system. And this reflects that the complex of MIT-ssDNA is more stable despite it has fewer binding sites. Therefore, we can conclude that MIT tends to react with ssDNA comparing with dsDNA. ${ }^{35}$

Under the same conditions as in the dsDNA and ssDNA systems, the spectra of two oligonucleotides systems were shown in Figure 5 (linear regression equations of $\alpha, \beta$ system are $Y=408.81 X+54.34$, $Y=114.68+47.78 X)$. From the figure, we can see that RLS intensity of oligonucleotides $\alpha$ system largely increases with the increase of the concentration of oligonucleotides $\alpha$. But the change of oligonucleotides $\beta$ concentration leads to little change of RLS intensity of the system. In order to describe the DNA bases recognition of MIT clearly, $K_{\mathrm{RLS}}$ and $N$ of these two systems were also studied according to the equations above. For these two oligonucleotides, $K_{\mathrm{RLS}}$ and $N$ of these two systems were also shown in Table 1. From the results, it's obvious that although with a smaller $N, K_{\mathrm{RLS}}$ of oligonucleotides $\alpha$ system is larger than that of oligonucleotides $\beta$, which displays that it is easier for the reaction between MIT and oligonucleotides $\alpha$. Therefore, we can conclude that MIT has a preference to the sequence, which is abundant with GC bases. ${ }^{36}$

Relative researches have confirmed that the anthraquinone ring of MIT can insert into the duplex of DNA. Meanwhile, the hydrogen bond between the electronegative groups of MIT and DNA can stabilize the MIT-DNA complex. From the results above, it's obvious that MIT tends to react with ssDNA compared with dsDNA. We deduce that the selectivity is mainly resulted from the steric effect of dsDNA. In other words, the duplex of dsDNA blocks the inserting of MIT molecule. As the decrease of torsional strain of ssDNA, it is easier for the formation of hydrogen bond between MIT and ssDNA. As a kind of weak interaction mode, hydrogen bond has an important role in assembling of macromolecules and molecular recognitions. Therefore, we consider it's the presence of these hydrogen bonds that induces the DNA sequences recognition of MIT. As to the bases selectivity of MIT, the results in this work are coincident with the latest research. ${ }^{37,38}$ Additionally, we consider that the two long flexible chains of MIT can spread to the opposite direction, respectively, and form hydrogen bonds with the amino of guanine and cytosine. Thus, MIT has an obvious preference to oligonucleotides $\alpha$.

In this contribution, DNA sequence recognition of MIT as well as its selectivity mechanisms was studied non-damagingly by a RLS strategy. The advantage of high sensitivity of RLS technique got full played in this work. From the results above, it can be seen that MIT tends to interact with ssDNA spontaneously and has selectivity to GC sequences. And their equilibrium constants and binding numbers were also
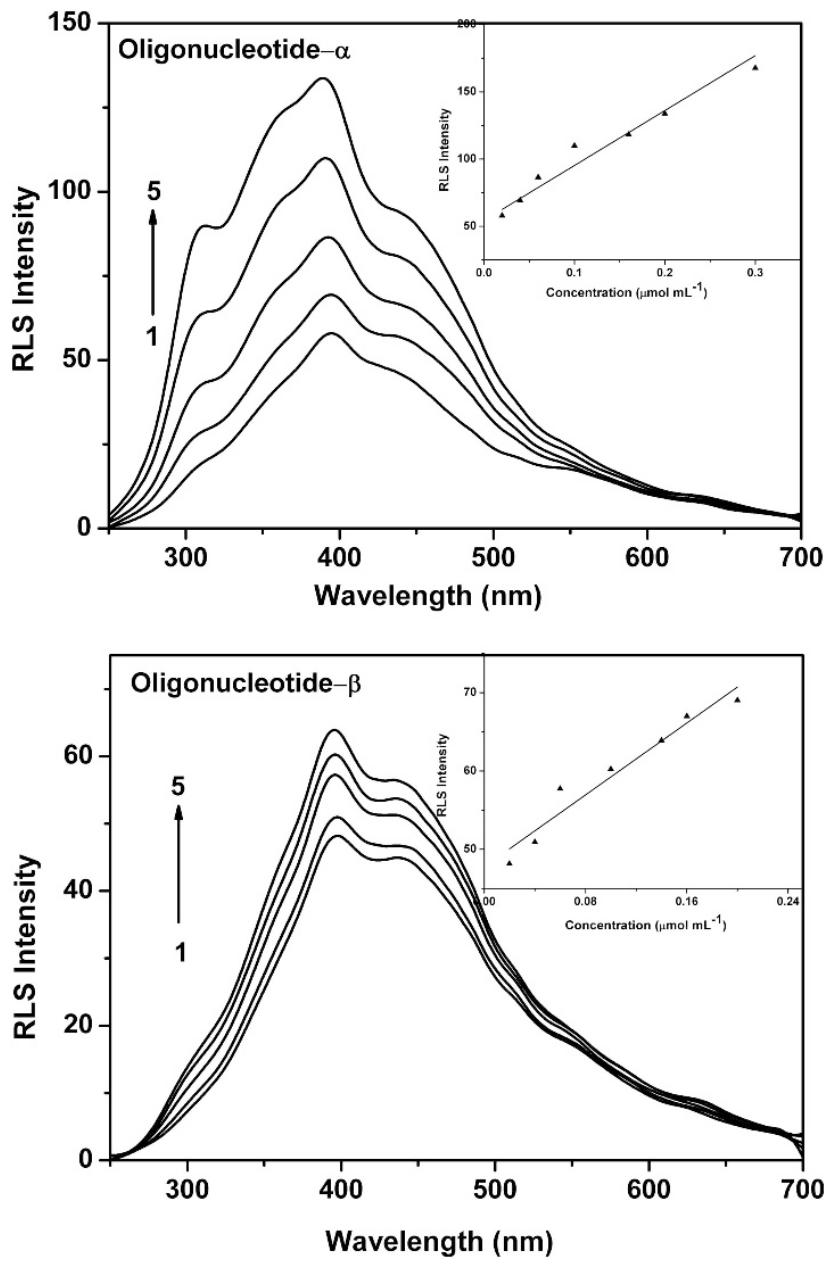

Figure 5 The RLS spectrum of MIT-oligonucleotides. (The inset is the relationship between the RLS intensity and concentration of oligonucleotides) Conditions: $\mathrm{pH}=2.0, \mathrm{MIT}, 1.0 \mu \mathrm{g} \mathrm{ml}-1$, oligonucleotides $\alpha$ oligonucleotides $\beta\left(\mu \mathrm{mol} \mathrm{ml}^{-1}\right)$ : $1-0.02,2-0.04,3-0.06,4-0.10$, $5-0.16$.

\begin{tabular}{|c|c|c|c|}
\hline & Regression equation between $1 / n$ and $1 /[\mathrm{L}]$ & $\mathrm{K}_{R L S}$ & $\mathrm{~N}$ \\
\hline dsDNA & $Y=68.99 X+801.48$ & $11.62 \mathrm{mmol} \mathrm{mg}^{-1}$ & $1.25 \times 10^{-3} \mathrm{mmol} \mathrm{mg}^{-1}$ \\
\hline ssDNA & $Y=76.01 X+1152.46$ & $15.16 \mathrm{mmol} \mathrm{mg}^{-1}$ & $8.68 \times 10^{-4} \mathrm{mmol} \mathrm{mg}^{-1}$ \\
\hline Oligonucleotides $\alpha$ & $Y=4.86 X+83.69$ & $17.291 \mathrm{mmol}^{-1}$ & $1.19 \times 10^{-2} / \mathrm{mmol}^{-1}$ \\
\hline Oligonucleotides $\beta$ & $Y=2.58 X+38.63$ & $14.97 \mathrm{Immol}^{-1}$ & $2.59 \times 10^{-2} / \mathrm{mmol}^{-1}$ \\
\hline
\end{tabular}

Table 1 The interaction parameters of MIT in different systems

Abbreviations: dsDNA, double-stranded DNA; MIT, mitoxantrone; RLS, resonance light scattering; ssDNA, single-stranded DNA. 
calculated. Compared with other methods, the RLS strategy displayed the real direct interaction between anticancer drugs and DNA in vitro without the influence of a foreign substance. Also in this work, the advantages of high sensitivity and simplicity of the RLS strategy indicated that it would be an essential method in DNA sequence recognition studies of DTAD. Moreover, the results of this work not only have verified the latest research achievements about MIT, but also can supply a theoretical basis for the improvements of the selectivity of anticancer drugs and the similar anticancer drugs design.

\section{EXPERIMENTAL PROCEDURE}

\section{Materials and Reagents}

MIT and dsDNA (calf thymus DNA, ctDNA) were all purchased from Sigma Company, St Louis, MO, USA; MIT solution $\left(20.0 \mu \mathrm{g} \mathrm{ml}^{-1}\right)$ was obtained by dissolving certain amount MIT in doubly distilled water and stocked in dark. The stock solution of dsDNA was prepared by dissolving the commercial products. The concentration of working solution of dsDNA was $20.0 \mu \mathrm{g} \mathrm{ml}^{-1}$. The ssDNA was obtained by treatment of DNA above $90^{\circ} \mathrm{C}$ for $15 \mathrm{~min}$ and then chilling in ice-water bath. Two oligonucleotides $\left(\alpha\right.$ : $5^{\prime}$-GGCCGGCC GCGCGGCCGGCC-3', $\beta: 5^{\prime}$-AATTAATTATATAATTAATT- $\left.3^{\prime}\right)$ were synthesized by Beijing Sunbiotech Co., Ltd. (Beijing, China) and their working concentration was $2.0 \mu \mathrm{moll}^{-1}$. Britton-Robinson (BR) buffer solution was used to adjust the $\mathrm{pH}$ of solutions. Doubly distilled water was used throughout. And all chemicals used without other illustration were of analytical grade or the best grade commercially available. All above solutions need to be stored at $4{ }^{\circ} \mathrm{C}$.

\section{Apparatus}

Both RLS spectra and fluorescence spectra were measured on a Perkin-Elmer LS-55 spectrofluoremeter equipped with a $1 \mathrm{~cm} \times 1 \mathrm{~cm}$ quartz cuvette. The AFM schemes were gotten on a MultiMode Nanoscope IIIa atomic force microscopy (Digital Instrument Company). All pH measurements were made with a SA720 Instrument (Orion Research, Toronto, ON, Canada).

\section{Experimental procedure}

Into a 10-ml calibrated flask were added certain amount of BR buffer, MIT solution and DNA. The prepared solution was diluted to $10.0 \mathrm{ml}$ with doubly distilled water and stirred thoroughly waiting for determination. The RLS spectra were studied by scanning simultaneously the excitation and emission monochromators $(\Delta \lambda=0)$ from 250.00 to $700.00 \mathrm{~nm}$. Both excitation and emission slits were $10.0 \mathrm{~nm}$. In the fluorescence assay, the preparation of solutions was similar as in the RLS spectra method. And the emission fluorescence spectra were scanned with $\lambda_{\mathrm{ex}}=608$ and $5.0 \mathrm{~nm}$ slit.

AFM samples were prepared by combining the advantages of spin-coating method and cationic deposition method. First, the new dissociated mica sheets have to be washed by $1.0 \mathrm{mmoll}^{-1} \mathrm{MgSO}_{4}$ in order to improve their loading capacity and $20 \mu \mathrm{l}$ prepared solutions were dripped on the micas and then the micas were put on the center of a centrifuge making the solutions overspread the micas under slow rotating speed. After 1-2 min deposition, $20 \mu \mathrm{l}$ doubly distilled water was used to wash the samples by the same method above. At last, after nature airing, the samples were scanned on a MultiMode Nanoscope IIIa atomic force microscopy.

\section{ACKNOWLEDGEMENTS}

The authors gratefully acknowledge financial support from the National Natural Science Foundation of China (39670298) and the Science Foundation of Shantou University (YR09009).

1 Wu, C. C. et al. Structure basis of Type II topoisomerase inhibition by the anticancer drug etoposide. Science 22, 459-462 (2011).

2 Rauf, S., Nawaz, H., Akhtar, K., Ghauri, M. A. \& Khalid, A. M. Studies on sildenafil citrate (Viagra) interaction with DNA using electrochemical DNA biosensor. Biosens. Bioelectron. 22, 2471-2477 (2007)
3 Wang, L. P., Lin, L. \& Ye, B. X. Electrochemical studies of the interaction of the anticancer herbal drug emodin with DNA. J. Pharmaceut. Biomed. Anal. 42, 625-629 (2006).

4 Hotze, G., Kariuki, B. M. \& Hannon, M. J. Dinuclear, double-stranded metallosupramolecular ruthenium complexes: potential anticancer drugs. Angew. Chem. 118, 4957-4960 (2006).

5 Gossens, C., Tavernelli, I. \& Rothlisberger, U. DNA structural distortions induced by Ruthenium -Arene anticancer compounds. J. Am. Chem. Soc. 130, 10921-10928 (2008).

$6 \mathrm{Siu}$, F. M. \& Che, C. M. Persistence of camptothecin analog-Topoisomerase I-DNA ternary Complexes: a molecular dynamics study. J. Am. Chem. Soc. 130, 17928-17937 (2008).

7 Halamikova, A., Vrana, O., Kasparkova, J. \& Brabec, V. Biochemical studies of the thermal effects on DNA modifications by the antitumor cisplatin and their repair. ChemBioChem 8, 2008-2015 (2007).

8 Tao, Z. M., Jones, E., Goodisman, J. \& Souid, A. K. Quantitative measure of cytotoxicity of anticancer drugs and other agents. Anal. Biochem. 381, 43-52 (2008).

9 Ricardo, P. T. Multidrug resistance: retrospect and prospects in anti-cancer drug treatment. Curr. Med. Chem. 13, 1859-1876 (2003).

10 Carrasco, C. et al. Tight binding of the antitumor drug ditercalinium to quadruplex DNA. ChemBioChem 3, 1235-1241 (2002).

11 Saad, J. S., Natile, G. L. \& Marzilli, G. Fundamental NMR solution studies utilizing right-handed cross-link models having $5^{\prime}$ - and $3^{\prime}$-Flanking residues. J. Am. Chem. Soc. 131, 12314-12324 (2009).

12 Takahara, P. M., Frederick, C. A. \& Lippard, S. J. Crystal structure of the anticancer drug cisplatin bound to duplex DNA. J. Am. Chem. Soc. 118 12309-12321 (1996).

13 Deubel, D. V. The chemistry of dinuclear analogues of the anticancer drug Cisplatin. A DFT/CDM study. J. Am. Chem. Soc. 128, 1654-1663 (2006).

14 Yuan, J. P., Guo, W. W., Yang, X. R. \& Wang, E. K. Anticancer drug-DNA interactions measured using a photoinduced electron-transfer mechanism based on luminescent quantum dots. Anal. Chem. 81, 362-368 (2009).

15 Rauf, S. et al. Electrochemical approach of anticancer drugs-DNA interaction. J. Pharmaceut. Biomed. Anal. 37, 205-217 (2005).

16 Pasternack, R. F. Bustamante, C. Collings, P. J., Giannetto, A \& Gibbs, E. J. Porphyrin assemblies on DNA as studied by a resonance light-scattering technique. J. Am. Chem. Soc. 115, 5393-5399 (1993)

17 Pasternack, R. F. \& Collings, P. J. Resonance light scattering: A new technique for studying chromophore aggregation. Science 269, 935-959 (1995).

18 Huang, C. Z., Li, K. A. \& Tong, S. Y. Determination of nucleic acids by a Resonance Light-Scattering technique with $r$, $\hat{a}, c ̧, a ̈$-Tetrakis [4-(trimethylammoniumyl) phenyl] porphine. Anal. Chem. 68, 2259-2263 (1996).

19 Ling, J. et al. Light- scattering signals from nanoparticles in biochemical assay, pharmaceutical analysis and biological imaging. Trac-Trend Anal. Chem. 28, 447-453 (2009).

20 Brar, S. K. \& Verma, M. Measurement of nanoparticles by light-scattering techniques. Trac-trend Anal. Chem. 30, 4-17 (2011).

21 Guo, H. Q., Cai, C. Q., Gong, H. \& Chen, X. M. Multi-spectroscopic method study the interaction of anti-inflammatory drug ketoprofen and calf thymus DNA and its analytical application. Spectrochem. Acta A 79, 92-96 (2011).

22 Chen, Z. G., Peng, Y. R., Chen, J. H. \& Zhu, L. Determination of antibacterial quaternary ammonium compound in lozenges and human serum by resonance light scattering technique. J. Pharmaceut. Biomed. Anal. 48, 946-950 (2008).

23 Ling, J., Li, Y. F. \& Huang, C. Z. A label-free visual immunoassay on solid support with silver nanoparticles as plasmon resonance scattering indicator. Anal. Biochem. 383, 168-173 (2008).

$24 \mathrm{Li}$, L., Song, G. W. \& Fang, G. R. Determination of bovine serum albumin by a resonance light-scattering technique with the mixed-complex $\mathrm{La}(\mathrm{Phth})(\text { phen })^{3+}$. J. Pharmaceut. Biomed. Anal. 40, 1198-1201 (2006).

25 Jiang, Z. L., Huang, Y. J., Liang, A. H., Pan, H. C. \& Liu, Q. Y. Resonance scattering detection of trace microalbumin using immunonanogold probe as the catalyst of Fehling reagent-glucose reaction. Biosens. Bioelectron. 24, 1674-1678 (2009).

26 Chen, Z. G. et al. Determination of anionic surfactant in surface water by resonance light-scattering technology. Int. J. Environ. Anal. Chem. 90, 573-585 (2009).

27 Chen, Z. G. et al. A new way to detect the interaction of DNA and anticancer drugs based on the decreased resonance light scattering signal and its potential application. Analyst 136, 3927-3933 (2011).

28 Chen, Z. G., Peng, Y. R., Chen, M. H., Chen, X. \& Zhang, G. M. DNA as a target for anticancer compounds screening directly by resonance light scattering technique. Analyst 135, 2653-2660 (2010)

29 Chen, Z. G. et al. Screen anticancer drug in vitro using resonance light scattering technique. Talanta 77, 1365-1369 (2009).

30 Chen, Z. G. et al. Screening DNA-targeted anticancer drug in vitro based on the drugconjugated DNA by resonance light scattering technique. Biosens. Bioelectron. 25, 1947-1952 (2010)

31 Riahi, S. et al. A theoretical study on interactions between Mitoxantrone as an anticancer drug and DNA: application in drug design. Chem. Biol. Drug Des. 71, 474-482 (2008).

32 Kostjukov, V. V., Pahomov, V. I., Andrejuk, D. D., Davies, D. B. \& Evstigneev, M. P. Investigation of the complexation of the anti-cancer drug novantrone with the hairpin structure of the deoxyheptanucleotide 50-d(GpCpGpApApGpC). J. Mol. Struct. 843, 78-86 (2007). 
33 Zhang, J. H., Umemoto, S. \& Nakatani, K. Fluorescent indicator displacement assay for legend-RNA interactions. J. Am. Chem. Soc. 132, 3660-3661 (2010).

34 Zhang, M. L., Sheng, G. P. \& Yu, H. Q. Determination of proteins and carbohydrates in the effluents from wastewater. Water Res 42, 3464-3472 (2008).

35 Brett, M. O., Macedo, T. R. A., Raimundo, D., Marques, M. H. \& Serrano, S. H. P. Voltammetric behaviour of mitoxantrone at a DNA-biosensor. Biosens. Bioelectron. 13, 861-867 (1998).
36 Lown, J. W., Morgan, A. R., Yen, S. F. \& Wang, Y. H. Characteristics of the binding of the anticancer agents mitoxantrone and ametantrone and related structures to deoxyribonucleic acids. Biochemistry 24, 4028-4035 (1985).

37 Wang, S. F., Peng, T. Z. \& Yang, C. F. Electrochemical determination of interaction parameters for DNA and mitoxantrone in anirreversible redox process. Biophys. Chem. 104, 239-248 (2003).

38 Kelley, S. O., Jackson, N. M., Hill, M. G. \& Barton, J. K. Long-range electron transfer through DNA films. Angew. Chem. 111, 991-996 (1999).

Supplementary Information accompanies the paper on The Journal of Antibiotics website (http://www.nature.com/ja) 